\title{
Nodular pseudotumoral pulmonary amyloidosis mimicking pulmonary carcinoma
}

\begin{abstract}
A 72 year old woman was admitted for a cholecystectomy. The preoperative chest radiograph revealed an incidental pulmonary density in the left upper lobe. Her medical history was non-contributory. She was a nonsmoker and had neither pulmonary nor systemic symptoms. A CT scan showed a $5 \mathrm{~cm}$ diameter non-calcified mass in the left upper lobe (fig 1A). Mediastinal lymphadenopathy was not present. Positron emission tomography with ${ }^{18} \mathrm{~F}-\mathrm{FDG}$ (FDG-PET) showed that the mass had a high heterogeneous uptake suggestive of malignancy (fig lB). A small left hilar satellite lesion and a high uptake mediastinal lesion compatible with metastasis were also detected. There was no evidence of contralateral or distant high uptake lesions. Fibreoptic bronchoscopy revealed no endobronchial lesion. The patient underwent a left superior lobectomy with mediastinal lymphadenectomy. Macroscopic examination of the left upper lobe showed a firm pale tanned and regular
\end{abstract}
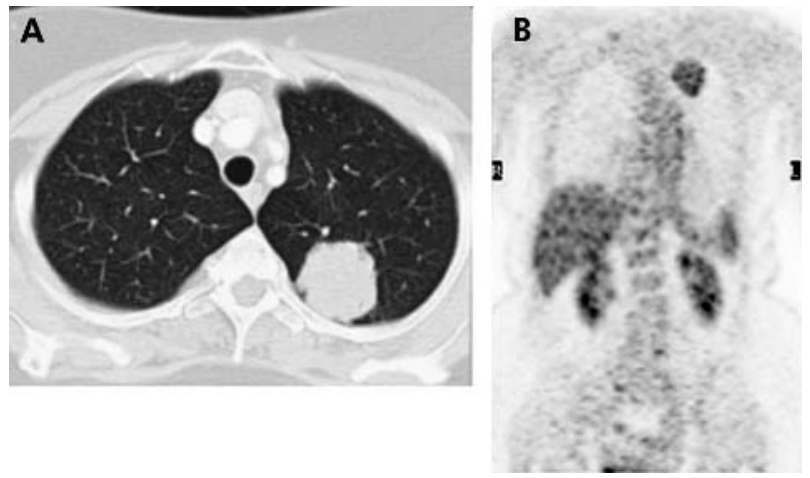

Figure 1 (A) CT scan showing lesion enhanced with contrast. (B) FDGPET scan showing lesion in the right upper lobe.

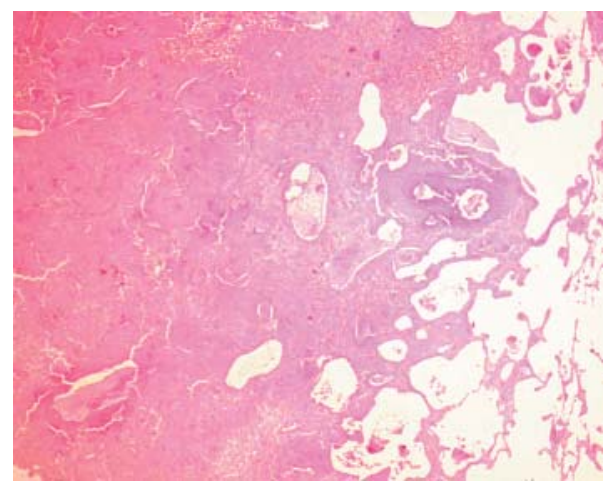

Figure 2 Histology of the amyloid nodule.

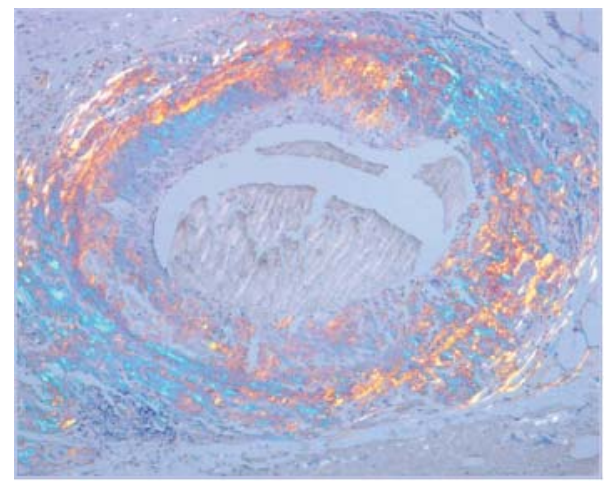

Figure 3 Amyloid birefringence under polarising microscopy.

subpleural nodule with well defined borders $(5.1 \times 4.7 \times$ $3.7 \mathrm{~cm}$ ). Histologically, the nodule was composed of a dense accumulation of eosinophilic, amorphous, homogeneous material, sharply demarcated from the surrounding lung tissue (fig 2). No carcinoma was seen. Congo Red staining gave an orange colour with apple green birefringence under polarising microscopy, features pathognomonic of amyloidosis (fig 3).

It is noteworthy that the amyloid nodule showed significant FDG activity on PET scan. FDG-PET is widely accepted as an important diagnostic tool in evaluating patients with possible cancers; it is not tumour-specific and a number of non-malignant processes can result in increased FDG accumulation. ${ }^{12}$ FDG-PET is a sensitive diagnostic modality for metabolically active lesions. Its positivity in this lesion is thus surprising, given the sparse nature of the inflammatory infiltrate. One case of pulmonary amyloidosis positive on PET has been reported in the literature. Despite its rarity, pulmonary nodular amyloidosis also forms part of the differential diagnosis of pulmonary opacities and coin lesions with high FDG uptake on PET. CT guided transbronchial biopsy can be a valuable tool in the diagnosis of nodular amyloidosis, avoiding unnecessary surgical resections. ${ }^{3}$

M Pusztaszeri, E M Kamel, S Artemisia, M Genevay, T McKee Pathology, Nuclear Medicine, and Diagnostic and Interventional Radiology Divisions, CHUV, Lausanne, Switzerland

Correspondence to: $\operatorname{Dr} M$ Pusztaszeri, Institut universitaire de Pathologie, 1011 Lausanne, Switzerland; Marc.Pusztaszeri@hospvd.ch

\section{REFERENCES}

1 Kung J, Zhuang $\mathrm{H}, \mathrm{Yu} J \mathrm{~J}$, et al. Intense fluorodeoxyglucose activity in pulmonary amyloid lesions on positron emission tomography. Clin Nucl Med 2003;28:975-6

2 Asad S, Aquino SL, Piyavisetpat N, et al. False positive FDG positron emission tomography uptake in non malignant chest abnormalities. AJR 2004; 182:983-9.

3 Kitamura H, Kobayashi T, Kaneko M, et al. Pulmonary amyloidosis diagnosed by CT-guided transbronchial biopsy: a case report. Jpn J Clin Oncol 2001;31:209-11. 